\title{
Molecular theory of glyphosate adsorption to pH-responsive polymer layers
}

\author{
Néstor A. Pérez-Chávez ${ }^{1}$. Alberto G. Albesa' · Gabriel S. Longo ${ }^{1}$
}

Received: 27 June 2018 / Revised: 12 February 2019 / Accepted: 17 April 2019 / Published online: 26 April 2019

(c) Springer Science+Business Media, LLC, part of Springer Nature 2019

\begin{abstract}
By means of a molecular-level theory we investigate glyphosate adsorption from aqueous solutions to surface-grafted poly(allylamine) layers. Our molecular model of glyphosate and the polymeric material includes description of size, shape, conformational freedom, and state of protonation of both components. The composition of the bulk solution ( $\mathrm{pH}$, salt concentration and glyphosate concentration) plays a critical role to determine adsorption. Adsorption is a non-monotonic function of the solution $\mathrm{pH}$, which can be explained in terms of the $\mathrm{pH}$-dependent protonation behavior of both adsorbate and adsorbent material. Lowering the solution salinity is an efficient way to enhance glyphosate adsorption. This is because glyphosate and salt anions compete for adsorption to the polymer layer. In this competition, glyphosate deprotonation, to increase its negative charge upon entering the polymer layer, plays an critical role to favor its adsorption under a variety of solution conditions. This deprotonation is the result of the higher $\mathrm{pH}$ that establishes inside the polymer. Our results show that such $\mathrm{pH}$ increase can be controlled, while achieving significant glyphosate adsorption, through varying the grafting density of the material. This result is important since glyphosate degradation by microbial activity is $\mathrm{pH}$-dependent. These polymeric systems are excellent candidates for the development functional materials that combine glyphosate sequestration and in situ biodegradation.
\end{abstract}
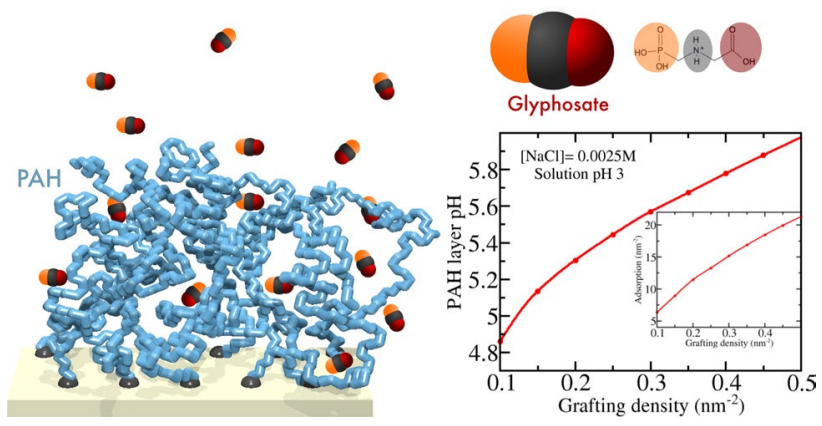

Keywords Glyphosate $\cdot$ Adsorption $\cdot$ Molecular theory

\section{Introduction}

Glyphosate (N-[phosphonomethyl] glycine) is the top-selling, most extensively applied pesticide worldwide (Woodburn 2000; Benbrook 2016). In Argentina, glyphosate

Gabriel S. Longo

longogs@inifta.unlp.edu.ar

1 Instituto de Investigaciones Fisicoquímicas Teóricas y Aplicadas (INIFTA), UNLP-CONICET, La Plata, Argentina commercialization and use has continuously grown since first introduced more than three decades ago (Primost et al. 2017). In general, this broad spectrum herbicide is used nonselectively for weed control, particularly in no-till farming practices (Soracco et al. 2018). Glyphosate-based herbicides work by blocking the shikimic acid pathway used by plants for the biosynthesis of aromatic amino acids, causing plant death by amino acid starvation (Martinez et al. 2018). However, glyphosate efficiency as an herbicide, its presumed non-toxicity to humans, and the development of 
crops genetically modified to resist glyphosate exposure, have led to its excessive application, which has resulted in negative effects, damaging the very same crops where applied (Martinez et al. 2018) and the surrounding fauna (Lopes et al. 2018; Burella et al. 2018; Sharma et al. 2018).

Glyphosate has been found in soils, groundwaters, and surface waters including its bottom sediments (Ronco et al. 2016; Rendon-von Osten and Dzul-Caamal 2017; Castro Berman et al. 2018). For example, the Lujan River in Argentina contains more than $3000 \mu \mathrm{g} / \mathrm{kg}$ of glyphosate (Primost et al. 2017; Castro Berman et al. 2018). In soils, it accumulates at a rate of $1 \mathrm{mg}$ glyphosate/kg soil every five spraying events (Primost et al. 2017), which can lead to an important degree of environmental pollution. Glyphosate has been detected in drinking water and other products of daily use (Younes and Galal-Gorchev 2000; World Health Organization 2005). Therefore, development of means for glyphosate removal must become an essential issue of present-day technology.

Methods of glyphosate removal currently include chemical precipitation (Liu et al. 2013), advanced oxidation (Lan et al. 2016; Xing et al. 2018), membrane filtration (Song et al. 2013), biodegradation (Loperena et al. 2007), and physical adsorption (Zhou et al. 2017; Santos et al. 2017; Guo et al. 2018; Zavareh et al. 2018). Glyphosate adsorption to resins (Zhou et al. 2017), graphene oxides (Santos et al. 2017), starch (Guo et al. 2018), and zeolites (Zavareh et al. 2018) has been investigated. Removal methods involving physical adsorption are generally efficient and more environmentally friendly (Zhou et al. 2017); however, because these methods are generally based on electrostatic attractions between the substrate and the herbicide, adsorption is highly affected by the solution composition. Several studies highlight the importance of $\mathrm{pH}$ and salt concentration in the adsorption of glyphosate to different materials (Zhou et al. 2017; Guo et al. 2018; Zavareh et al. 2018).

Therefore, understanding the role of solution composition in modulating the electrostatic interactions is important when designing new materials for glyphosate removal through physical adsorption. In this work, we investigate glyphosate adsorption from aqueous salt solutions to a surface-grafted poly(allylamine) (PAH) layer. We are particularly interested in the effect of solution composition (salt concentration and $\mathrm{pH}$ ) on the degree of glyphosate adsorption to this material. To achieve this goal, we apply a molecular theory that accounts for size, shape and conformation of glyphosate as well as those of the polymeric substrate. In this method, the state of protonation of glyphosate titratable units and that of allylamine segments is not assumed a priori depending on the $\mathrm{pH}$, but rather they are predicted depending on the local conditions that are consistent with the global thermodynamic equilibrium. Our results suggest that PAH-based polymeric materials can be used in the development of functional materials for glyphosate sequestration.

Degradation of glyphosate in soils and waters occurs as a result of microbial activity (Hallas et al. 1992; la Cecilia and Maggi 2018). The enzymatic activity of the bacteria responsible for glyphosate degradation slows down when the environment $\mathrm{pH}$ is not neutral. This behavior under nonneutral conditions reduces biodegradation efficiency and leads to longer glyphosate persistence in the environment (la Cecilia and Maggi 2018). Our work shows that the grafting density of the PAH layer can be used as a design parameter to enhance glyphosate sequestration while simultaneously tune the environment inside the material for optimal in situ biodegradation.

\section{Methods}

To investigate the equilibrium adsorption of glyphosate to grafted PAH layers, we apply a theoretical approach that accounts specifically for the molecular details of all chemical species in the system. This molecular theory describes size, shape, configuration, and local charge state of both glyphosate and the polymer layer. Namely, the protonation state of each of the different titratable units of glyphosate is not assumed but predicted as a result of the local conditions that yield thermodynamic equilibrium. For this, we formulate a general thermodynamic potential that includes the free energy cost of protonation/deprotonation, the entropic loss of molecular confinement, the conformational degrees of freedom of both polymer layer and adsorbate, and the electrostatic and steric interactions. This theory is an extension of the method developed by Nap et al. (2006) and Gong et al. (2007) to study grafted weak polyelectrolyte layers. Next, we outline the most important details of the method while a more complete description is presented in Appendix.

The system of study is composed of $N$ surface-grafted poly(allylamine) chains in contact with an aqueous glyphosate solution (see Fig. 1). The coordinate $z$ gives the distance from the planar supporting surface, which is placed at $z=0$ and has total area $A$; then, PAH grafting density is $\sigma=\frac{N}{A}$. The solution contains water molecules, hydronium and hydroxyde ions, a monovalent salt $(\mathrm{NaCl})$, completely dissociated into chloride and sodium ions, and glyphosate.

The Helmholtz free energy of this system can be expressed as

$$
\begin{aligned}
F= & -T S_{\text {conf }}-T S_{\text {mix }}-T S_{g l y} \\
& +F_{\text {chm,pol }}+F_{c h m, g l y}+U_{\text {ste }}+U_{\text {elec }}
\end{aligned}
$$

where $T$ is the system temperature, $S_{\text {conf }}$ is the configurational entropy of polymer chains, and $S_{m i x}$ is the translational 




Fig. 1 Scheme representing the system of study. A surface-grafted poly(allylamine hydrochloride) layer is in contact with an aqueous glyphosate solution. This scheme also illustrates the coarse-grained model used to describe glyphosate molecule and the polymer

(mixing) entropy of mobile chemical species (excluding glyphosate). The chemical free energy of the polymer chains and that of glyphosate are $F_{c h m, p o l}$ and $F_{c h m, g l y}$, respectively. These contributions describe the acid-base equilibria of the different titratable units of each component. The energetic contributions are the steric repulsions (excluded-volume), $U_{\text {ste }}$, and the electrostatic interactions, $U_{\text {elec }}$.

Far from the surface the composition of the bulk solution is externally controlled, through imposing the bulk $\mathrm{pH}$, and the concentrations of salt $([\mathrm{NaCl}])$ and glyphosate ([gly]); these quantities are the independent variables of our calculations. This implies that the chemical potentials of all free (mobile) species are the same in different regions of the system, including inside the polymer layer and in the solution both close and far from the polymer-solution interface. Under such conditions, the proper thermodynamic potential whose minimum yields equilibrium is the semi-grand potential, which is a function of these chemical potentials. This thermodynamic potential results from the Legendre transform of the Helmholtz free energy.

All different physicochemical contributions to the free energy, Eq. (1), can be explicitly written in terms of a few functions: (i) the probability distribution of PAH conformations; (ii) the local densities of all free species, including that of different conformations (rotations) of glyphosate; (iii) the local degrees of protonation of all titratable species including PAH and glyphosate units; and (iv) the local electrostatic potential. Optimization of the semi-grand potential with respect to each of these functions leads to expressions for functions (i), (ii) and (iii) in terms of only two local interaction potentials: the local osmotic pressure and the electrostatic potential. Such local interaction potentials are evaluated at each position through calculating the numerical solution of the Poisson equation and the mathematical constraint resulting the incompressibility of the fluid system. This procedure is more extensively described in Appendix. After both interaction potentials have been determined, the free energy is known and any equilibrium quantity of interest can be derived. We emphasize again that this optimization of the system's thermodynamic potential includes the different degrees of freedom of all molecular components.

In order to calculate results using this theory, a molecular model of the different chemical species must be specified. In particular, the different conformations of a PAH chain are an input of the method. These chains are 100-segment long chains, where each segment is a coarse-grained representation of a PAH unit, having $0.5 \mathrm{~nm}$ segment length, and volume $v_{A H}=0.065 \mathrm{~nm}^{3}$. Then, we are modeling a grafted layer of $9.3 \mathrm{kDa} \mathrm{PAH}$. A large set of conformations of these polymer chains are generated using a Rotational Isomeric State (RIS) model (Flory 1969), where each polymer segment can assume one out of three iso-energetic relative orientations. The pKa of the allylamine group is 9.86 (PérezMitta et al. 2018).

The molecular model of glyphosate molecule is a coarsegrained representation that includes its three main groups: phosphonate, amine and carboxylate group. The molecular volumes assigned to these coarse-grained units are $v_{p h p}=0.065 \mathrm{~nm}^{3}, v_{a m n}=0.045 \mathrm{~nm}^{3}$, and $v_{c b x}=0.045 \mathrm{~nm}^{3}$, respectively. The pKa values of these units are 0.78 and 2.27 for the diacidic phosphonate group, 5.58 for the carboxylic acid, and 10.25 for the basic amine group (Chamberlain et al. 1996). The atomic coordinates of glyphosate molecule have been obtained through density functional theory (DFT) (Kohn and Sham 1965) geometry optimizations using Gaussian 03 package (Frisch et al. 2003), where we have applied the B3LYP hybrid exchange-correlation functional (Lee et al. 1988; Becke 1993) and the 6-31G(d,p) basis set. In these calculations, all geometrical parameters have been optimized without constraints; we consider the isolated glyphosate molecule without including the solvent or the polymer network. The coarse-grained units are assigned to the position of the Carbon atom in the carboxylate group, the Nitrogen in the amine, and the Phosphorus in the phosphonate group. These positions are kept fixed with respect to the center of mass of the glyphosate molecule according to the DFT optimization of the atomistic structure. The molecule, however, has full rotational and translational freedom.

Other input values required in our calculations are the volume of water molecules and its ions $\left(0.030 \mathrm{~nm}^{3}\right)$, and the volume of chloride and sodium ions $\left(0.033 \mathrm{~nm}^{3}\right)$. For the self-dissociation of water we use $\mathrm{pK}_{w}=14$. In order to solve the equations that result from the molecular theory (i.e., the Poisson equation and the incompressibility constraint), the volume of the system is divided into $0.5 \mathrm{~nm}$-thick layers parallel to the supporting surface (the $x-y$ plane). These 


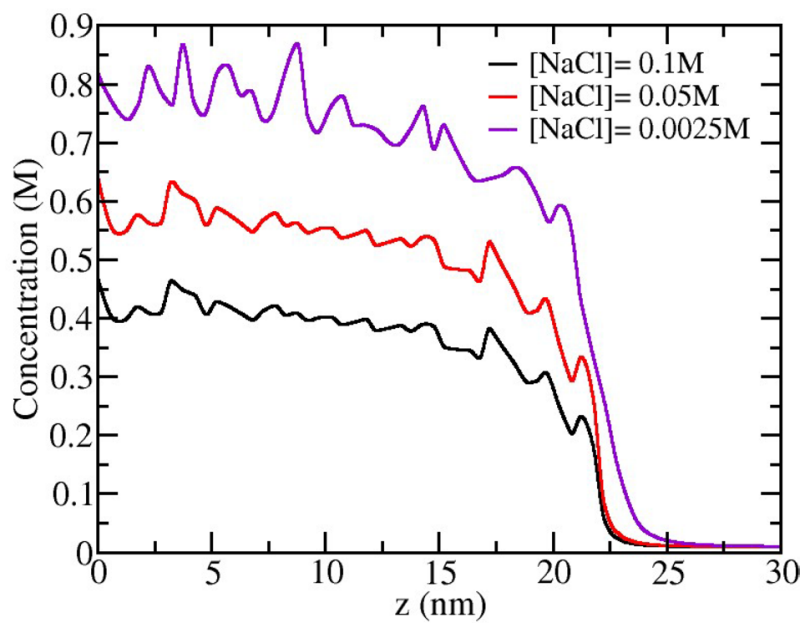

Fig. 2 Glyphosate concentration as a function of distance to the supporting surface (placed at $z=0$ ); three different salt concentrations are shown at $\mathrm{pH} 7$ and $[\mathrm{gly}]=0.01 \mathrm{M}$

equations are solved using a Jacobian-free Newton-Krylov method. We assume that the system is isotropic in the $x-y$ plane. The dielectric constant of the medium is $\epsilon_{w} \epsilon_{0}$, where $\epsilon_{w}=78.5$ is the relative dielectric constant of water at room temperature and $\epsilon_{0}$ is the permittivity of vacuum.

\section{Results and discussion}

The theory applied in this work allows for calculating the local densities of all chemical species that are consistent with thermodynamic equilibrium of the system. To illustrate this, Fig. 2 shows glyphosate concentration as a function of the distance to the supporting surface, under different environmental conditions. Three clearly distinctive spatial regions can be observed in this graph: sufficiently far from the supportive surface ( $z \gtrsim 25 \mathrm{~nm}$ ), the concentration of glyphosate is that corresponding to the bulk solution $([\mathrm{gly}]=0.01 \mathrm{M})$. Inside the polymer layer, which extends to $z \approx 20 \mathrm{~nm}$, a higher concentration establishes with respect to the bulk solution, which indicates adsorption. In particular, a larger concentration of glyphosate adsorbs as the salinity of the solution decreases. At intermediate distances, the concentration of glyphosate decreases as $z$ increases from the value inside the polymer to the bulk concentration. The thickness of this interfacial region, as seen in the examples of Fig. 2, depends on the salt concentration.

Figure 2 shows that the composition of the solution influences both glyphosate concentration inside polymer film and the thickness of the polymer-solution interfacial region. To further quantify this behavior, we define the adsorption, $\Gamma$, as the number of molecules adsorbed in excess of the bulk contribution,

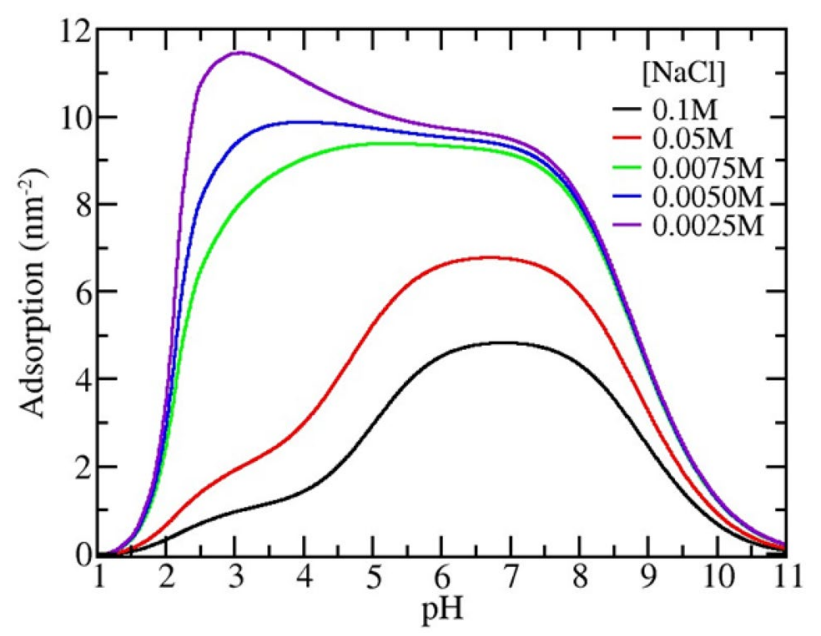

Fig. 3 Adsorption of glyphosate as a function of the solution $\mathrm{pH}$. Curves for different salt concentrations are included. PAH grafting density is $\sigma=0.2 \mathrm{~nm}^{-2}$, and bulk glyphosate concentration is [gly $]=0.01 \mathrm{M}$

$\Gamma=\int_{0}^{\infty} d z\left(\left\langle\rho_{g l y}(z)\right\rangle-\rho_{g l y}^{b u l k}\right)$

where $\left\langle\rho_{g l y}(z)\right\rangle$ and $\rho_{g l y}^{b u l k}$ are respectively the local and bulk number density of glyphosate. Angle brackets in the local density indicate ensemble average over molecular conformations; these quantities satisfy $\lim _{z \rightarrow \infty}\left\langle\rho_{g l y}(z)\right\rangle=\rho_{g l y}^{\text {bulk }}$.

Figure 3 presents the dependence of the adsorption on the composition of the bulk solution ( $\mathrm{pH}$ and salt concentration). As seen in Fig. 2, glyphosate adsorption decreases with increasing solution salinity. This behavior is a clear indication that glyphosate adsorption is driven by electrostatic attractions with the positively charged PAH layer, and evidences glyphosate competition with chlorine anions for adsorption. As the bulk concentration of salt increases, a larger number of these ions partition inside the layer, resulting in the screening of PAH-glyphosate attractions, which diminishes adsorption. Indeed, for the cases shown in Fig. 2, the concentration of adsorbed chloride ions is $0.027,0.45$ and $0.75 \mathrm{M}$ for $[\mathrm{NaCl}]=0.0025,0.05$, and 0.1 $\mathrm{M}$, respectively.

Another feature seen in Fig. 3 is that glyphosate adsorption is a non-monotonic function of the solution $\mathrm{pH}$, independently of salt concentration. To explain this behavior, Fig. 4 shows the net (total) charge of adsorbed and solution glyphosate as a function of $\mathrm{pH}$ for different salt concentrations; the inset of this graph presents the degree of charge of polymer segments at the same conditions. At sufficiently low $\mathrm{pH}$, the polymer layer is strongly, positively charged as seen in this inset. Under these conditions however, solution glyphosate is also positively charged, and 


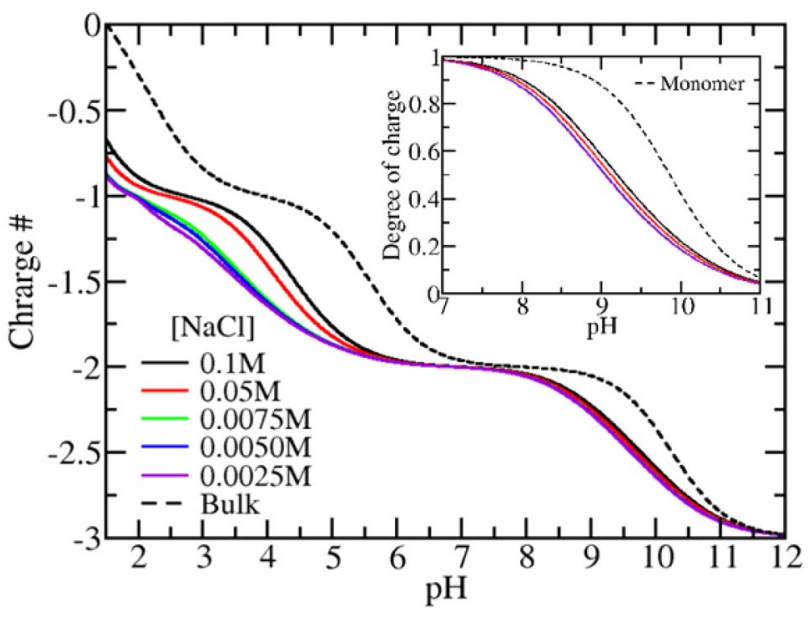

Fig. 4 Plot of net electric charge of adsorbed (solid-line curves, corresponding to different salt conditions) and solution (dashed-line curve) glyphosate molecules as a function of $\mathrm{pH}$. Inset shows the degree of charge of PAH segments under the same conditions (solidline curves); as a reference, this inset also includes the degree of charge of the isolated PAH monomer (dashed line); $\sigma=0.2 \mathrm{~nm}^{-2}$ and [gly $]=0.01 \mathrm{M}$

adsorbed glyphosate only bears a weak negative net charge (as compared to chloride ions), which explains the negligible adsorption at very acidic conditions seen in Fig. 3. In the other side of the $\mathrm{pH}$ scale, both adsorbed and solution glyphosate are strongly negatively charged (see Fig. 4). In this case, however, the polymer layer is weakly charged, leading to negligible adsorption for highly alkaline conditions. For intermediate values of $\mathrm{pH}$, on the other hand, both glyphosate and the polymer are strongly and oppositely charged, which results in significant adsorption and explains the non-monotonic behavior seen in Fig. 3.

The two main features of the behavior presented in Fig. 3 have been experimentally reported for glyphosate adsorption to other materials. For example, adsorption from aqueous solutions to cross-linked amino-starch decreases with increasing $\mathrm{NaCl}$ concentration, and it displays a nonmonotonic dependence on $\mathrm{pH}$ (Guo et al. 2018). Such pHdependent behavior has also been reported for glyphosate adsorption to zeolites (Zavareh et al. 2018) and nano-sized microporous exchange resins (Zhou et al. 2017). In these different systems, glyphosate adsorption is driven by electrostatic attractions with the adsorbent material; the considerations that we have presented to describe how the behavior depends on solution composition can also be applied to those systems.

Figure 4 shows that glyphosate becomes more negatively charged with respect to the bulk solution upon adsorption to the polymer layer. This charge regulation behavior occurs to enhance the electrostatic attractions with the polymer that drive adsorption; charge regulation results from a lower

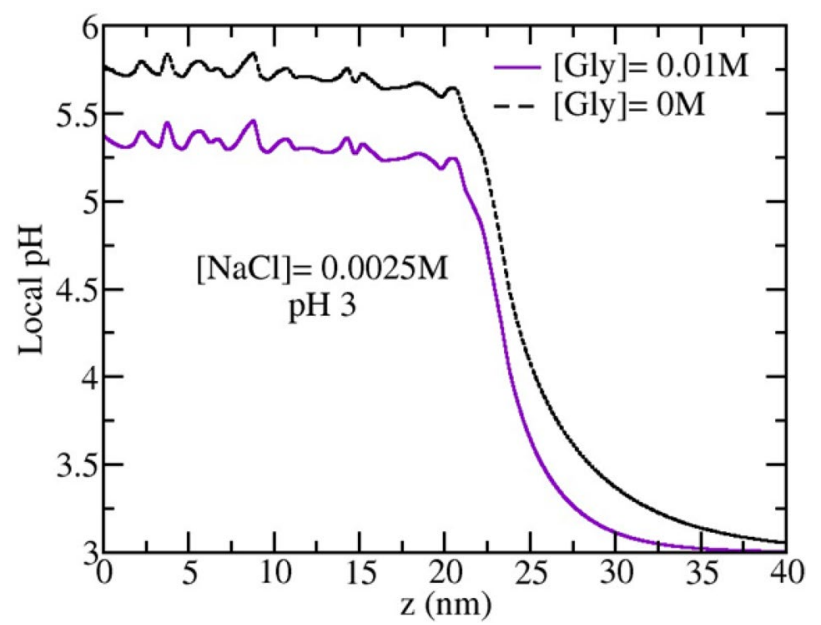

Fig. 5 Plot showing local $\mathrm{pH}$ as a function of the distance to the grafting surface for bulk solutions with (solid line) and without (dashed line) glyphosate; $\sigma=0.2 \mathrm{~nm}^{-2}$

degree of protonation of those titratable units of glyphosate inside the PAH layer. This phenomenon occurs in the whole $\mathrm{pH}$ scale, but it is more accentuated at acidic conditions (see Fig. 4). In our molecular model, the three pKa values of glyphosate acidic units (carboxylate and phosphonate) occur in this $\mathrm{pH}$ region. Thus, in order to favor adsorption, the molecule has greater flexibility to regulate charge under such acidic conditions. In addition, the charge of adsorbed glyphosate depends on the salt concentration (see Fig. 4). Upon adsorption at acidic conditions, the lower the bulk concentration of salt, the more negatively charged glyphosate is.

Glyphosate regulates its charge because the $\mathrm{pH}$ increases near and inside the polymer with respect to the bulk solution, which allows its titratable units to have a higher local degree of deprotonation resulting in a more negative net charge. To illustrate this change in local pH, Fig. 5 shows this quantity as a function of the distance to the grafting surface. We define the local $\mathrm{pH}$ as

$\mathrm{pH}(z)=-\log _{10}\left[H^{+}\right](z)$

where $\left[\mathrm{H}^{+}\right](z)$ is the local concentration of hydronium ions. In particular, we concentrate in the conditions that display maximum adsorption in Fig. 3, pH3 and $0.0025 \mathrm{M} \mathrm{NaCl}$. Local $\mathrm{pH}$ presents the same three regions as the local concentration of glyphosate (compare Figs. 2 and 5): far from the surface $(z \gg 25 \mathrm{~nm})$, local $\mathrm{pH}$ approaches the bulk $\mathrm{pH}$; the polymer-solution interfacial region extends significantly longer for the bulk solution without glyphosate. The lowsalt conditions of Fig. 5 means that electrostatic interactions extend roughly $12-15 \mathrm{~nm}$ from the top surface of the polymer layer. In contrast, the presence of glyphosate in relatively high concentration increases the ionic strength of 
the solution, which reduces the effective reach of these interactions. As a consequence the interface extends significantly less, 8-10 $\mathrm{nm}$ approximately.

Moreover, Fig. 5 shows that inside the polymer layer a higher $\mathrm{pH}$ establishes $(z \lesssim 20 \mathrm{~nm})$. This higher $\mathrm{pH}$ accounts for the charge regulation behavior seen in Fig. 4. Interestingly, glyphosate presence leads to a lower $\mathrm{pH}$ inside the polymer with respect to solutions without this adsorbate. For salt solutions without glyphosate, this increase in $\mathrm{pH}$ occurs to induce deprotonation of PAH units and reduce segment-segment electrostatic repulsions. For a solution containing glyphosate, $\mathrm{pH}$ in this region lowers, which allows for the PAH to be more protonated (than in the absence of the adsorbate). Such response increases the positive charge of the polymer and favor the electrostatic attractions with the adsorbate. However, should this drop in $\mathrm{pH}$ not occur, glyphosate would be even more negatively charged, which could potentially strengthen these attractions as well; we remind the reader that adsorbed glyphosate is more negatively charged (less protonated) than in the bulk solution.

Therefore, the $\mathrm{pH}$ that establishes inside the polymer layer (and in other regions of the system as well) is the consequence of the complex balance of between all physicochemical contributions to the free energy put simultaneously into play to determine the thermodynamic equilibrium at the environmental conditions externally imposed (bulk $\mathrm{pH}$, salt and glyphosate concentrations). In this context, the local $\mathrm{pH}$ is a useful quantity that allows for knowing the state of protonation/charge of all species in the vicinity. In particular the $\mathrm{pH}$ inside the polymer layer provides such information for both adsorbate and adsorbent material. Then, to further characterize this behavior, we define $\mathrm{pH}_{\mathrm{pol}}$ as the position average of the local $\mathrm{pH}$ inside the polymer layer

$\mathrm{pH}_{\mathrm{pol}}=\frac{1}{h} \int_{0}^{h} d z \mathrm{pH}(z)$

where $\mathrm{pH}(z)$ is the local $\mathrm{pH}$ (see Eq. (3)), and $h$ is the polymer layer thickness.

Figure 6 present the polymer film $\mathrm{pH}$ at the same salt conditions of Fig. 5. With respect to the bulk solution, $\mathrm{pH}_{\mathrm{pol}}$ is higher in most of the $\mathrm{pH}$ range, particularly under acidic conditions. At bulk $\mathrm{pH} 3$, for example, the $\mathrm{pH}$ is more than 2 units larger inside the polymer layer. As we mentioned, this bulk $\mathrm{pH}$ corresponds to the maximum adsorption shown in Fig. 3. For more neutral and alkaline conditions, $\mathrm{pH}_{\mathrm{pol}}$ is significantly lower than for a salt solution without glyphosate.

This change in $\mathrm{pH}$ inside the polymer layer is important if one is to design new materials that combine glyphosate sequestration with in situ degradation. Several field studies have shown that microbial degradation of glyphosate is optimal when the $\mathrm{pH}$ is close to neutral (Yu et al. 2015;

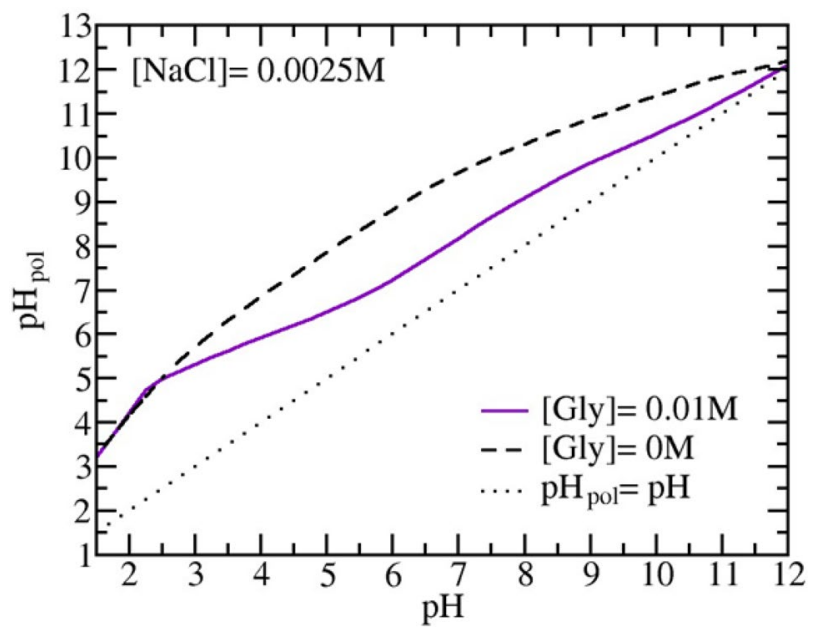

Fig. 6 Plot of $\mathrm{pH}$ inside the polymer layer, $\mathrm{pH}_{\mathrm{pol}}$, for solutions with (solid line) and without (dashed line) glyphosate. Dotted line illustrate bulk (ideal) conditions where $\mathrm{pH}_{\mathrm{pol}}=\mathrm{pH} ; \sigma=0.2 \mathrm{~nm}^{-2}$

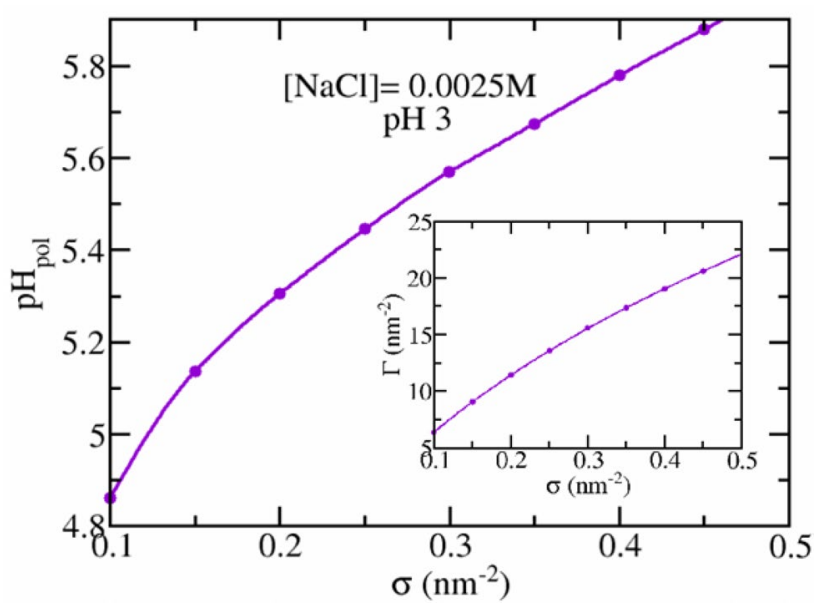

Fig. 7 Plot of the $\mathrm{pH}$ inside the polymer as a function of the polymer grafting density for $0.01 \mathrm{M}$ glyphosate solutions. The lower-right inset shows the adsorption at the same conditions

Manogaran et al. 2017; la Cecilia and Maggi 2018). In this context, we ask the question: is it possible to obtain significant adsorption to the PAH layer while at the same achieve the right conditions inside for biodegradation? In field experiments, the only design parameter is the grafting density of the polymer, since the $\mathrm{pH}$ and salt concentrations are determined by the environment. Therefore, Fig. 7 shows the $\mathrm{pH}$ inside the polymeric material as a function of the grafting density. The solution conditions that we have chosen are those consistent with previous figures ( $\mathrm{pH} 3$, $0.0025 \mathrm{M} \mathrm{NaCl}$ and $0.01 \mathrm{M}$ glyphosate). The $\mathrm{pH}$ of the polymer layer increases with grafting density as does the adsorption of glyphosate, which is shown in the lower rightcorner of Fig. 7. These results suggest that depending on the 
environment conditions the grafting density can be used as a design parameter in order to combine significant adsorption and enhanced in-material biodegradation.

\section{Conclusions}

We have applied a theoretical approach to study the adsorption of glyphosate from aqueous solutions to a surface-grafted poly(allylamine) layer. As experimental results have shown for other materials where adsorption is driven by electrostatic interactions, our results show that solution composition plays a critical role in the adsorption behavior. In particular, lowering the solution salinity is the best way to enhance adsorption, given the environment glyphosate concentration. This is because glyphosate and salt anions compete for adsorption to the polymer layer. In other words, increasing the salt concentration screens the polymer-glyphosate electrostatic attractions.

Adsorption depends non-monotonically on the solution $\mathrm{pH}$. This behavior results from the protonation behavior of both the polymer and glyphosate: the polymer is uncharged at sufficiently high $\mathrm{pH}$, while the adsorbate bears either a positive, a neutral, or a small negative average charge at low $\mathrm{pH}$. Only at intermediate values of $\mathrm{pH}$, both components are strongly and oppositely charged to result in adsorption.

Glyphosate deprotonates upon adsorption to the polymer layer, which increases its negative charge and favors adsorption to the positively charged polymeric material. This charge-regulation behavior occurs in the whole range of $\mathrm{pH}$ values, but it is more relevant at acidic conditions. In our glyphosate model, the pKa of its acidic units correspond to low $\mathrm{pH}$ values, which gives the molecule greater flexibility to deprotonate under such conditions to enhance interactions with the polymer chains. This charge-regulation is of key importance because otherwise no adsorption should occur for acidic solutions where glyphosate bears less negative charge than a chloride ion.

This charge regulation upon adsorption occurs because the $\mathrm{pH}$ that establishes inside the polymer layer is significantly higher than in the solution. This increase in local $\mathrm{pH}$, however, means that PAH segments are more weakly charged than one isolated monomer would be in a solution at the same conditions. This behavior occurs to reduce polymer-polymer repulsions. Upon glyphosate adsorption, PAH units are more positively charged as compared with solutions without glyphosate (but still less charged than the isolated monomer).

Finally, our results show that the $\mathrm{pH}$ inside the polymer layer can be turned by changing PAH grafting density. This is important because bacterial degradation of glyphosate is $\mathrm{pH}-$ dependent. Changing the grafting density allows for significant adsorption while controlling the $\mathrm{pH}$ inside the polymer layer.
Therefore, grafting density can be used as a key parameter in the design of polymeric materials with optimal sequestration/ degradation properties. We are currently conducting more systematic studies on the effect of grafting density as well as the influence of polymer molecular weight.

Acknowledgements This work was supported by CONICET and ANPCyT (Grant No. PICT-2014-3377), Argentina. N. A. P. C. acknowledges a ANPCyT fellowship (Grant No. PICT-2015-3425).

\section{Appendix}

\section{Theoretical approach}

In this section, we give expressions for the different contributions to the free energy, Eq. (1). The first term in the right-hand side of that equation contains the conformational entropy of the polymer layer,

$-\frac{S_{\text {conf }}}{k_{B}}=N \sum_{\alpha} P(\alpha) \ln (P(\alpha))$

where $P(\alpha)$ is the probability of finding a polymer chain in its conformation $\alpha, k_{B}$ is the Boltzmann constant, and $N$ is the number of chains grafted to the surface of total area $A$.

In the second contribution to the free energy, the translational (mixing) entropy of mobile species (except glyphosate) is

$$
\begin{aligned}
-\frac{S_{m i x}}{k_{B}}= & A \sum_{\gamma=w, H^{+}, O H^{-},+,-} \int_{0}^{\infty} d z \rho_{\gamma}(z) \\
& \times\left(\ln \left(\rho_{\gamma}(z) \nu_{w}\right)-1+\beta \mu_{\gamma}^{0}\right)
\end{aligned}
$$

where the subindex $\gamma$ runs over water molecules $(w)$, hydronium $\left(\mathrm{H}^{+}\right)$and hydroxyde ions $\left(\mathrm{OH}^{-}\right)$, salt cations $(+)$and anions $(-) ; \rho_{\gamma}(z)$ gives the local number density of the corresponding species; $v_{w}$ is the volume of a water molecule, and $\beta=\frac{1}{k_{B} T}$. This contribution also includes the standard chemical potential (self-energies) of each of these free species, $\mu_{\gamma}^{0}$.

The translational and configurational (rotational) entropy of the glyphosate molecule is

$$
\begin{aligned}
-\frac{S_{g l y}}{k_{B}}= & A \int_{0}^{\infty} d z \sum_{\alpha_{g l y}} \rho_{g l y}\left(\alpha_{g l y}, z\right) \\
& \times\left(\ln \left(\rho_{g l y}\left(\alpha_{g l y}, z\right) \nu_{w}\right)-1+\beta \mu_{g l y}^{0}\right)
\end{aligned}
$$

where $\rho_{g l y}\left(\alpha_{g l y}, z\right)$ is the local density of glyphosate in conformation $\alpha_{g l y}$. This contribution also contains the 
self-energy of the molecule, given by its standard chemical potential $\mu_{g l y}^{0}$. Then, the total density of glyphosate at $z$ is

$\left\langle\rho_{g l y}(z)\right\rangle=\sum_{\alpha_{g l y}} \rho_{g l y}\left(\alpha_{g l y}, z\right)$

where \langle\rangle indicate ensemble average over molecular conformations.

Next term in Eq. (1) is the chemical free energy that describes the acid-base equilibrium of PAH units. This contribution can be expressed as

$$
\begin{aligned}
\beta F_{\text {chm }, p o l}= & N \int_{0}^{\infty} d z\langle\rho(z)\rangle f_{A H}(z) \\
& \times\left(\ln f_{A H}(z)+\beta \mu_{A H p}^{0}\right) \\
& +N \int_{0}^{\infty} d z\langle\rho(z)\rangle\left(1-f_{A H}(z)\right) \\
& \times\left(\ln \left(1-f_{A H}(z)\right)+\beta \mu_{A H d}^{0}\right)
\end{aligned}
$$

where $f_{A H}(z)$ is the local degree of charge (or protonation) of PAH units, and $\langle\rho(z)\rangle$ is the ensemble average density of segments belonging to a single chain, such that

$$
\langle\rho(z)\rangle=\sum_{\alpha} P(\alpha) \rho(\alpha, z)
$$

with $\rho(\alpha, z)$ being the local density of segments of a chain in conformation $\alpha$. In Eq. (9), $\mu_{A H p}^{0}$ and $\mu_{A H d}^{0}$ are the standard chemical potentials of the protonated and deprotonated unit, respectively. These quantities are related to the thermodynamic equilibrium constant, $K_{A H}^{0}$, through the following equation:

$K_{A H}^{0}=\exp \left(\beta \mu_{A H p}^{0}-\beta \mu_{A H d}^{0}-\beta \mu_{H^{+}}^{0}\right)$

The chemical free energy of glyphosate, which accounts for the acid-base equilibria of its titratable units is

$$
\begin{aligned}
\beta F_{c h m, g l y}= & \sum_{\tau} A \int_{0}^{\infty} d z\left\langle\rho_{\tau}(z)\right\rangle \\
& \times g_{\tau}(z)\left(\ln g_{\tau}(z)+\beta \mu_{\tau p}^{0}\right) \\
& +\sum_{\tau} A \int_{0}^{\infty} d z\left\langle\rho_{\tau}(z)\right\rangle\left(1-g_{\tau}(z)\right) \\
& \times\left(\ln \left(1-g_{\tau}(z)\right)+\beta \mu_{\tau d}^{0}\right)
\end{aligned}
$$

where $\tau$ runs over the different titratable units of glyphosate, which are the diacidic phosphonate group, the carboxylate group and the amine group $\left(\tau=p h p_{1}, p h p_{2}, c b x, a m n\right)$; the standard chemical potentials of these units are $\mu_{\tau p}^{0}$ and $\mu_{\tau d}^{0}$ for the protonated and deprotonated unit, respectively. The local degree of protonation of $\tau$ units is $g_{\tau}(z)$. In the case of the amine group, this quantity is equal to its local degree of charge $f_{\text {amn }}(z)$, while for the acidic units $f_{\tau}(z)=1-g_{\tau}(z)$ (with $\tau=p h p_{1}, p h p_{2}, c b x$ ). In addition, the local density of glyphosate units can be expressed as:

$$
\begin{aligned}
\left\langle\rho_{\tau}(z)\right\rangle= & \sum_{\alpha_{g l y}} \int_{0}^{\infty} d z^{\prime} \rho_{g l y}\left(\alpha_{g l y}, z^{\prime}\right) \\
& \times n_{\tau}\left(\alpha_{g l y}, z^{\prime}, z\right)
\end{aligned}
$$

where $n_{\tau}\left(\alpha_{g l y}, z^{\prime}, z\right)$ is the number (density) of units type $\tau$ that a glyphosate molecule with center of mass at $z^{\prime}$ contributes to $z$.

The next term in Eq. (1) is $U_{\text {ste }}$, which describes the steric repulsions at the excluded volume level. This contribution is incorporated through requiring each element of volume to be fully occupied by some of the chemical species. This constraint to the the free energy can be expressed as:

$$
\begin{aligned}
1=N\langle\rho(z)\rangle \nu_{A H} & +\sum_{\gamma=w, H^{+} O H^{-},+,-} \rho_{\gamma}(z) v_{\gamma} \\
& +\sum_{\tau=a m n, c b x, p h p}\left\langle\rho_{\tau}(z)\right\rangle \nu_{\tau}
\end{aligned}
$$

where $v_{A H}$ is the volume of a PAH segment, $v_{\gamma}$ is that of free species $\gamma$, and $v_{\tau}$ the volume of glyphosate unit $\tau$.

The last contribution to $F$ is the electrostatic energy,

$$
\begin{aligned}
U_{\text {elec }}= & A \int_{0}^{\infty} d z\left[\left\langle\rho_{q}(z)\right\rangle \beta \psi(z)\right. \\
& \left.-\frac{1}{2} \beta \epsilon(\nabla \psi(z))^{2}\right]
\end{aligned}
$$

where $\epsilon$ is the medium permittivity, and $\psi(z)$ is the positiondependent electrostatic potential. In this equation, the local density of charge is

$$
\begin{aligned}
\left\langle\rho_{q}(z)\right\rangle=N f_{A H}(z)\langle\rho(z)\rangle q_{A H} & +\sum_{\gamma=H^{+} O H^{-},+,-} \rho_{\gamma}(z) q_{\gamma} \\
& +\sum_{\tau=p h p_{1}, p h p_{2}, c b x, a m n} f_{\tau}(z)\left\langle\rho_{\tau}(z)\right\rangle q_{\tau}
\end{aligned}
$$

$q_{A H}, q_{\gamma}$ and $q_{\tau}$ are the electric charges of the different species.

This system is in equilibrium with a bulk solution of controlled composition, which fixes the chemical potentials of all free species $\left(\mu_{\gamma}\right.$ and $\left.\mu_{g l y}\right)$. Under these conditions, the proper thermodynamic potential whose minimum yields equilibrium is the semi-grand potential, which can be written as 


$$
\begin{aligned}
\Omega= & F-\sum_{\gamma=w, H^{+} O H^{-},+,-} \mu_{\gamma} N_{\gamma}-\mu_{g l y} N_{g l y} \\
= & F-A \sum_{\gamma=w, H^{+} O H^{-},+,-} \int_{0}^{\infty} d z \mu_{\gamma} \rho_{\gamma}(z) \\
& -A \int_{0}^{\infty} d z \mu_{g l y}\left\langle\rho_{g l y}(z)\right\rangle
\end{aligned}
$$

where $N_{\gamma}$ and $N_{g l y}$ are the corresponding number of molecules.

With all these expressions for its different contributions, the grand potential can expressed in terms of integrals and configurational sums the following functions:

(i) $P(\alpha)$;

(ii) $\rho_{w}(z), \rho_{H^{+}}(z), \rho_{O H^{-}}(z), \rho_{+}(z), \rho_{-}(z), \rho_{g l y}\left(\alpha_{g l y}, z\right)$;

(iii) $f_{A H}(z), f_{\text {php }_{1}}(z), f_{\text {php }_{2}}(z), f_{a m n}(z), f_{c b x}(z)$;

(iv) $\psi(z)$.

Optimization of $\Omega$ with respect to functions (i) to (iii) leads to expressions for these functions that only depend on the two interaction potentials: $\psi(z)$ and $\pi(z)$, which is the Lagrange multiplier introduced to enforce satisfaction of the incompressibility constraint, Eq. (14). The extremum of $\Omega$ with respect to the electrostatic potential results in the Poisson equation:

$\frac{\partial^{2} \psi}{\partial z^{2}}=-\frac{\left\langle\rho_{q}(z)\right\rangle}{\varepsilon}$

Finally, $\psi(z)$ and $\pi(z)$ are obtained though iteratively solving the Poisson equation (Eq. (18)) and the incompressibility constraint (Eq. (14)) at each position. Once these interaction potentials have been calculated, functions $(i)$ to $(i v)$ are known, and consequently the free energy of the system is determined.

\section{References}

Becke, A.D.: Density-functional thermochemistry. III. The role of exact exchange. J. Chem. Phys. 98(7), 5648-5652 (1993)

Benbrook, C.M.: Trends in glyphosate herbicide use in the United States and globally. Environ. Sci. Eur. 28(1), 1-15 (2016)

Burella, P., Odetti, L., Simoniello, M., Poletta, G.: Oxidative damage and antioxidant defense in caiman latirostris (broad-snouted caiman) exposed in ovo to pesticide formulations. Ecotoxicol. Environ. Saf. 161, 437-443 (2018)

Castro Berman, M., Marino, D.J., Quiroga, M.V., Zagarese, H.: Occurrence and levels of glyphosate and AMPA in shallow lakes from the Pampean and Patagonian regions of Argentina. Chemosphere 200, 513-522 (2018)
Chamberlain, K., Evans, A.A., Bromilow, R.H.: 1-octanol/water partition coefficient (kow) and pka for ionisable pesticides measured by a ph-metric method. Pestic. Sci. 47(3), 265-271 (1996)

Flory, P.J.: Statistical Mechanics of Chain Molecules. Interscience, New York (1969)

Frisch, M., Trucks, G., Schlegel, H., Scuseria, G., Robb, M., Cheeseman Jr., J., Vreven, T., Kudin, K., Burant, J., Millam, J., Iyengar, S., Tomasi, J., Barone, V., Mennucci, B., Cossi, M., Scalmani, G., Rega, N., Petersson, G., Nakatsuji, M., Pople, J.: Gaussian 03, Revision B.03. Gaussian, Inc., Pittsburgh (2003)

Gong, P., Genzer, J., Szleifer, I.: Phase behavior and charge regulation of weak polyelectrolyte grafted layers. Phys. Rev. Lett. 98, 018302 (2007)

Guo, L., Cao, Y., Jin, K., Han, L., Li, G., Liu, J., Ma, S.: Adsorption characteristics of glyphosate on cross-linked amino-starch. J. Chem. Eng. Data 63(2), 422-428 (2018)

Hallas, L.E., Adams, W.J., Heitkamp, M.A.: Glyphosate degradation by immobilized bacteria: field studies with industrial wastewater effluent. Appl. Environ. Microbiol. 58(4), 1215-1219 (1992)

Kohn, W., Sham, L.J.: Self-consistent equations including exchange and correlation effects. Phys. Rev. 140(4A), 1133-1138 (1965)

la Cecilia, D., Maggi, F.: Analysis of glyphosate degradation in a soil microcosm. Environ. Pollut. 233, 201-207 (2018)

Lan, H., He, W., Wang, A., Liu, R., Liu, H., Qu, J., Huang, C.P.: An activated carbon fiber cathode for the degradation of glyphosate in aqueous solutions by the electro-fenton mode: optimal operational conditions and the deposition of iron on cathode on electrode reusability. Water Res. 105, 575-582 (2016)

Lee, C., Yang, W., Parr, R.G.: Development of the Colle-Salvetti correlation-energy formula into a functional of the electron density. Phys. Rev. B 37(2), 785-789 (1988)

Liu, Z., Zhu, M., Yu, P., Xu, Y., Zhao, X.: Pretreatment of membrane separation of glyphosate mother liquor using a precipitation method. Desalination 313, 140-144 (2013)

Loperena, L., Ferrari, M.D., Saravia, V., Murro, D., Lima, C., Ferrando, L., Fernández, A., Lareo, C.: Performance of a commercial inoculum for the aerobic biodegradation of a high fat content dairy wastewater. Bioresour. Technol. 98(5), 1045-1051 (2007)

Lopes, F.M., Sandrini, J.Z., Souza, M.M.: Toxicity induced by glyphosate and glyphosate-based herbicides in the zebrafish hepatocyte cell line (zf-1). Ecotoxicol. Environ. Saf. 162, 201-207 (2018)

Manogaran, M., Shukor, M.Y., Yasid, N.A., Johari, W.L.W., Ahmad, S.A.: Isolation and characterisation of glyphosate-degrading bacteria isolated from local soils in Malaysia. Rendiconti Lincei 28(3), 471-479 (2017)

Martinez, D.A., Loening, U.E., Graham, M.C.: Impacts of glyphosate-based herbicides on disease resistance and health of crops: a review. Environ. Sci. Eur. 30(1), 2 (2018)

Nap, R., Gong, P., Szleifer, I.: Weak polyelectrolytes tethered to surfaces: effect of geometry, acid-base equilibrium and electrical permittivity. J. Polym. Sci. Part B Polym. Phys. 44(18), 2638-2662 (2006)

Pérez-Mitta, G., Marmisollé, W.A., Albesa, A.G., Toimil-Molares, M.E., Trautmann, C., Azzaroni, O.: Phosphate-responsive biomimetic nanofluidic diodes regulated by polyamine-phosphate interactions: Insights into their functional behavior from theory and experiment. Small 14, 1702131 (2018)

Primost, J.E., Marino, D.J., Aparicio, V.C., Costa, J.L., Carriquiriborde, P.: Glyphosate and ampa,"pseudo-persistent" pollutants under real-world agricultural management practices in the mesopotamic pampas agroecosystem, argentina. Environ. Pollut. 229, 771-779 (2017)

Rendon-von Osten, J., Dzul-Caamal, R.: Glyphosate residues in groundwater, drinking water and urine of subsistence farmers from intensive agriculture localities: a survey in Hopelchén, 
Campeche, Mexico. Int. J. Environ. Res. Public Health 14(6), 595 (2017)

Ronco, A.E., Marino, D.J., Abelando, M., Almada, P., Apartin, C.D.: Water quality of the main tributaries of the Paraná basin: glyphosate and AMPA in surface water and bottom sediments. Environ. Monit. Assess. 188(8), 458 (2016)

Santos, T.R.T., Andrade, M.B., Silva, M.F., Bergamasco, R., Hamoudi, S.: Development of $\alpha$ - and $\gamma-\mathrm{Fe}_{2} \mathrm{O}_{3}$ decorated graphene oxides for glyphosate removal from water. Environ. Technol. 3330, 1-20 (2017)

Sharma, A., Jha, P., Reddy, G.V.: Multidimensional relationships of herbicides with insect-crop food webs. Sci. Total Environ. 643 , 1522-1532 (2018)

Song, J., Li, X.M., Figoli, A., Huang, H., Pan, C., He, T., Jiang, B.: Composite hollow fiber nanofiltration membranes for recovery of glyphosate from saline wastewater. Water Res. 47(6), 2065-2074 (2013)

Soracco, C.G., Villarreal, R., Lozano, L.A., Vittori, S., Melani, E.M., Marino, D.J.: Glyphosate dynamics in a soil under conventional and no-till systems during a soybean growing season. Geoderma 323, 13-21 (2018)

Woodburn, A.T.: Glyphosate: production, pricing and use worldwide. Pest Manag. Sci. 56(4), 309-312 (2000)
World Health Organization: glyphosate and AMPA in drinking-water. Technical Report. World Health Organization (2005)

Xing, B., Chen, H., Zhang, X.: Efficient degradation of organic phosphorus in glyphosate wastewater by catalytic wet oxidation using modified activated carbon as a catalyst. Environ. Technol. 39(6), 749-758 (2018)

Younes, M., Galal-Gorchev, H.: Pesticides in drinking water-A case study. Food Chem. Toxicol. 38, S87-S90 (2000)

Yu, X., Yu, T., Yin, G., Dong, Q., An, M., Wang, H., Ai, C.: Glyphosate biodegradation and potential soil bioremediation by Bacillus subtilis strain bs-15. Genet. Mol. Res. 14(4), 14717-14730 (2015)

Zavareh, S., Farrokhzad, Z., Darvishi, F.: Modification of zeolite 4A for use as an adsorbent for glyphosate and as an antibacterial agent for water. Ecotoxicol. Environ. Saf. 155, 1-8 (2018)

Zhou, C., Jia, D., Liu, M., Liu, X., Li, C.: Removal of glyphosate from aqueous solution using nanosized copper hydroxide modified resin: equilibrium isotherms and kinetics. J. Chem. Eng. Data 62(10), 3585-3592 (2017)

Publisher's Note Springer Nature remains neutral with regard to jurisdictional claims in published maps and institutional affiliations. 